\title{
"Beating osteoARThritis": Development of a stepped care strategy to optimize utilization and timing of non-surgical treatment modalities for patients with hip or knee osteoarthritis
}

\author{
Agnes J. Smink • Cornelia H. M. van den Ende • Thea P. M. Vliet Vlieland • \\ Bart A. Swierstra • Joke H. Kortland • Johannes W. J. Bijlsma • Theo B. Voorn • \\ Henk J. Schers • Sita M. A. Bierma-Zeinstra • Joost Dekker
}

Received: 13 May 2011 /Revised: 16 August 2011 / Accepted: 18 August 2011 /Published online: 2 September 2011

(C) Clinical Rheumatology 2011

\begin{abstract}
Inadequacies in health care practices have been reported despite existing guidelines to manage hip or knee osteoarthritis. To facilitate guideline implementation and improve utilization of non-surgical treatment options a care strategy should be developed. This study describes the development of an evidence-based, multidisciplinary, patient-centered, stepped care strategy. A national, multidisciplinary, steering group developed the strategy in three phases: (1) consensus among steering group members (first draft); (2) written consultation of 23 representatives of patient organizations and professional associations involved in osteoarthritis care (second draft); (3) consensus of the final draft after discussion in two rounds during a conference with representatives from the different disciplines. The final stepped care strategy presents, in three tiers, the optimal order for non-surgical treatment modalities. It
\end{abstract}

A. J. Smink $(\bowtie) \cdot$ C. H. M. van den Ende

Department of Rheumatology, Sint Maartenskliniek, P.O. Box 9011, 6500 GM Nijmegen, The Netherlands

e-mail: a.smink@maartenskliniek.nl

T. P. M. Vliet Vlieland

Department of Rheumatology and department of Orthopaedics, Leiden University Medical Center,

Leiden, The Netherlands

B. A. Swierstra

Department of Orthopaedics, Sint Maartenskliniek,

Nijmegen, The Netherlands

\section{J. H. Kortland}

Dutch Patient Organization for Rheumatic Diseases,

Amersfoort, The Netherlands recommends that more advanced options should only be considered if options listed in previous steps failed to produce satisfactory results. Hence, the first step treatment options can be offered to all patients but may also be provided through self care (education, life style advice, and acetaminophen). The second step (exercise therapy, dietary therapy, and non-steroidal anti-inflammatory drugs) and third step treatment options (multidisciplinary care, intra-articular injections, and transcutaneous electrical nerve stimulation) can be considered for people with persisting complaints. Trough a consensus procedure, we succeeded to develop a multidisciplinary, patient-centered, stepped care strategy based on national guidelines. This strategy provides a framework for health care providers and patients with hip or knee osteoarthritis to discuss the optimal timing of the various treatment options.

\section{J. W. J. Bijlsma}

Department of Rheumatology and Clinical Immunology,

University Medical Center,

Utrecht, The Netherlands

T. B. Voorn $\cdot$ H. J. Schers

Department of Primary and Community Care, Radboud

University Nijmegen Medical Center,

Nijmegen, The Netherlands

\section{S. M. A. Bierma-Zeinstra}

Department of General practice and department of Orthopaedics, Erasmus University Medical Center,

Rotterdam, The Netherlands

J. Dekker

Department of Rehabilitation Medicine,

VU University Medical Center,

Amsterdam, The Netherlands 
Keywords Hip osteoarthritis · Knee osteoarthritis . Patient-centered care $\cdot$ Practice guidelines

\section{Introduction}

As there is no known cure for osteoarthritis (OA), treatment is based on controlling pain, improving function, and improving health-related quality of life. The potential of the various non-surgical treatment modalities is clearly demonstrated in several national and international guidelines to manage hip or knee OA [1-5]. In general, a combination of pharmacological and non-pharmacological treatment modalities is considered to be the best core treatment. However, despite the availability of these guidelines inadequate diagnostic procedures, referrals, and utilization of treatment modalities are seen in clinical practice, leading to suboptimal outcomes.

A possible explanation for these inadequacies is the lack of recommendations in current guidelines about the indication for and the timing of treatment options, which over time can impede optimal OA management. This explanation is supported by the findings that only $28 \%$ of OA patients who are scheduled to undergo total hip arthroplasty had actually ever been referred to a physical therapist during their OA history [6], and that non-pharmacological treatment options, such as patient education, weight reduction and exercise therapy are underutilized [7-10].

Another possible explanation is the poor incorporation of guideline recommendations in clinical practice. For example, half of the primary care physicians ordered inappropriate diagnostic tests in a hypothetical patient with knee OA [9]. Furthermore, interventions lacking evidence for efficacy (e.g., massage, traction, and stretching) are still utilized in approximately half of the OA patients who received physiotherapy [11].

To facilitate guideline implementation and improve utilization of non-surgical treatment options, a multidisciplinary and patient-centered approach is suggested, as different health care providers are involved in the nonsurgical care of OA, and a central role of the patient in managing health in collaboration with providers is advocated [12-15]. This multidisciplinary and patient-centered approach could best be achieved by employing a "stepped" care model. A stepped care model provides a good framework for both health care providers as patients, as it presents the optimal order when a range of interventions is available and it uses limited resources to their greatest effect $[16,17]$. For these reasons, it appeared most suitable to manage a heterogenic population like OA patients for a prolonged period. The first step of such a model suggests interventions that should be tried initially, while interventions in later steps are reserved for those whose condition was not controlled by lower step treatment options. Thus, health care providers play an active role in monitoring and evaluating the previous performed treatment options. This model has been proven to be effective for several other chronic disorders, like diabetes mellitus [18], depression [19], and back pain [20].

To date, a few tools have been developed for health care providers to support decision making in the OA management. For instance, some clinical pathways describe diagnostic procedures and OA management [13], but lack recommendations about the timing for the different treatment modalities. While Porcheret et al. [21] did develop a stepped care strategy for older adults in primary care with knee pain or knee OA, there is no such comprehensive protocol to guide non-surgical management for patients with hip or knee OA.

In the Netherlands, therefore, several stakeholders took the initiative to develop a stepped care strategy [22], which takes into account the diverse presentation and progression of symptoms with the subsequent variation in treatment needs. This article describes the developmental process and the content of the multidisciplinary patient-centered, stepped care strategy for hip or knee OA, known as BART, i.e., Beating osteoARThritis.

\section{Methods}

The stepped care strategy was developed by a steering group in three phases. This steering group, appointed by the B\&JD NL board, consisted of a patient representative and six experts representing the main disciplines involved in OA care: rheumatology (TPMV), orthopaedic surgery (BAS), primary care (SMAB, TBV), physiotherapy (CHME) and rehabilitation (JD).

The first phase started with studying the content of national and international guidelines and performing a literature search to identify recently published systematic reviews about the management of OA, not incorporated in guidelines. The steering group discussed and defined, in several meetings, the starting points, in order to set a preliminary focus for the content of the stepped care strategy. Finally, the steering group formulated a first draft of the stepped care strategy on the basis of consensus after an open broad discussion in which differences were resolved by discussion.

In the second phase, 23 representatives of patient organizations and professional associations involved in OA care were asked to comment to a set of questions (see Table 1) on the first draft, by means of a written consultation (20 responded). These questions were used to aid the process of consensus by generating ideas for comments and suggestions. Subsequently, the steering 
Table 1 Questions presented during the written consultation with 23 representatives

1. Do you agree with the board of the B\&JD NL and the members of the national steering group that a more consistent and uniform management of the treatment for osteoarthritis is desirable?

2. Do you endorse the principles of the stepped care strategy created by the national steering group?

What is your opinion about this draft of stepped care strategy with regard to the following:

3. Is the stepped care strategy clearly formulated?

4. The stepped care strategy consist three steps. Do you think fewer or more steps are desirable?

5. Do you agree with the ranking of the treatment options?

6. Do you endorse the length of the evaluation period proposed for each step?

7. Do you want to add treatment options to this stepped care strategy?

8. Do you believe that specific treatment options mentioned in the stepped care strategy are not relevant? If so, which?

9. Do you believe that implementation of the stepped care strategy will indeed contribute to a more consistent and uniform management of the treatment of osteoarthritis?

10. Do you have other comments?

group summarised these comments and suggestions. Based on these results, a second draft of the stepped care strategy was made after differences were resolved by discussion.

Finally, during an invitational conference participants could give feedback on these results in two discussion rounds led by the chairman (TBV). Both rounds were performed by five panels. In the first round homogenous groups were made: one panel consisted of patient representatives and four panels of four different disciplines (general practitioners (GPs), physical therapists, orthopaedic surgeons and rheumatologists). After the first round the chairman made in a plenary session an inventory and summary of the main concerns and suggestions for change raised by the different panels. Then, those concerns and suggestions for change were discussed in a second round with five mixed panels. In the second plenary session, consensus was reached on the final version of the stepped care strategy.

\section{Developmental process}

\section{Starting points}

The steering group decided to build a three-step care strategy, each step consisting of recommendations for the timing of diagnostic procedures, treatment modalities, and evaluation. First, they reached consensus about the following starting points:

- The stepped care strategy is primarily based on the Dutch multidisciplinary guideline for the diagnosis and management of hip or knee OA [1]. While there is general consistency in the numerous recommendations among national and international guidelines as well as among monodisciplinary and multidisciplinary guidelines to manage hip or knee OA, recommendations do differ to some extent.
- The steering group acknowledged the importance of a patient-centered approach in the management of OA [23-25]. Therefore, the following elements were considered to be important and should be incorporated in the stepped care strategy: mutual goal setting, enhancing communication between health care providers and patient, facilitating an active role of the patient while being realistic about personal limitations as well as the availability of resources.

- The stepped care strategy presents in step 1 those interventions that should be offered (or achieved by self management) to all patients with hip or knee OA. Step 2 or 3 interventions should only be considered for patients with persisting pain or disability despite use of interventions from the lower step or steps.

- The stepped care strategy does not provide explicit statements indicating which specific professional should be involved, as some treatment options can be delivered by several health care providers. For example, education and life style advice can be provided by GPs, nurse practitioners, occupational therapists or physical therapists whereas intra-articular injections can be prescribed and administered by GPs as well as medical specialists.

\section{Discussions and decisions}

Comments offered during the different phases of the development process were discussed until consensus was reached. The major discussions and decisions pertaining to the three phases are described below.

- Some treatment modalities such as occupational therapy or braces are at the moment still commonly prescribed in clinical practice, although they are not recommended in evidence-based clinical guidelines. For that matter, the incorporation of these treatment modalities in the 
stepped care strategy was discussed. Nevertheless, it was decided that only treatment modalities that are recommended in evidence-based clinical guideless should be included.

- Contrary to the Dutch multidisciplinary guideline for the diagnosis and management of hip or knee OA, the international Osteoarthritis Research Society International (OARSI) guideline recommends the use of topical nonsteroidal anti-inflammatory drugs (NSAIDs) for knee OA based on a recent meta-analysis of randomized controlled trials (highest level of evidence, i.e., level 1a) [3]. Therefore, the stepped care strategy recommends the use of topical NSAIDs as a treatment modality for knee OA.

- The evidence that supports the use of glucosaminesulphate is not conclusive. The Dutch multidisciplinary guideline [1] advices glucosaminesulphate for a trial period, while the Dutch Guideline for General Practitioners [26, 27] clearly does not advice its use. Therefore, the stepped care strategy suggests the possibility of a trial period of 3 months, similar to the Dutch multidisciplinary guideline. The treatment should be discontinued if no response is apparent.

- The stepped care strategy should be concise and straightforward. Therefore, the strategy does not give recommendations concerning the dosage, assessment of contra-indications and prevention of side effects of pharmacological interventions (e.g., of NSAIDs), as well as those concerning the intensity and content of non-pharmacological interventions (e.g., physiotherapy).

- The monodisciplinary guideline of the Royal Dutch Society of Physical Therapy [28] advises restricted use of Transcutaneous Electrical Nerve Stimulation (TENS). So, only if exercise therapy and medication have not resulted in pain reduction, TENS should be recommended.

\section{Content of the stepped care strategy}

The final version of BART presents the optimal order of non-surgical treatment options for hip or knee OA in three steps.

Diagnostic procedures and assessment

No definitive test exists to diagnose OA. Therefore, the diagnosis is primarily based on medical history and physical examination. In step 2, the stepped care strategy recommends radiological assessment if there is a discrepancy between the medical history and physical examination.

Prior to mutual goal setting, health care providers are advised to assess the nature and the severity of loss in body functions and body structures as well as reduced activity and participation resulting from OA. Furthermore, in step 2 behavioural factors that might influence the symptoms and outcomes of treatment should be assessed.

Treatment modalities

The stepped care strategy implies that the more advanced options should only be considered if prior options failed. Therefore, treatment options in step 1, such as education (e.g., about the condition, treatment modalities and timing of care), lifestyle advice (e.g., about exercise, diet, and aids) and an adequate dose of acetaminophen, should be offered to all patients with OA. However, they can also be provided through self-care.

If the treatment options in step 1 are not sufficient, treatment options in step 2 can be considered: e.g., exercise therapy, NSAIDs, and tramadol, either alone or in combination, is recommended. Overweight patients, if motivated to lose weight, should be referred to a dietician.

Treatment options in step 3, should only to be considered if those in the previous steps were ineffective. They comprise the more advanced treatment options, e.g., multidisciplinary care, if available. Intra-articular injections with hyaluronic acid or glucocorticoids or TENS can be considered in patients with knee OA.

\section{Evaluation}

To optimize treatment results, mutual goal setting, systematic monitoring, evaluation, and if necessary, adjustment to the previously set goals are advocated.

In step 1, the effectiveness of the treatment should be evaluated after 3 months. Obviously, if the patient has persistent symptoms, evaluation can be performed earlier. In case of partial or non adherence to treatment modalities; patients' personal barriers should be identified, and possibilities to improve adherence should be discussed. In step 2, evaluation should occur after 3-6 months. In step 3, the evaluation moment should be planned with the patient. Depending on the setting, different health care providers such as a nurse practitioner could play an important role in evaluating and monitoring the treatment. A summary of all recommendations are shown in Table 2.

\section{Discussion}

BART provides a structured approach to achieve implementation of existing guidelines that emphasize self management and creates multidisciplinary support to manage OA. It provides a framework for OA patients and their health care providers to reduce inadequacies in the management of non-surgical treatment options, particularly 
Table 2 Summary of the recommendations in each step for the diagnostic procedures and assessment, treatment modalities, and evaluation

\begin{tabular}{|c|c|c|c|}
\hline & Step 1 & Step 2 & Step 3 \\
\hline $\begin{array}{l}\text { Diagnostic procedures } \\
\text { and assessment }\end{array}$ & $\begin{array}{l}\text { - Medical history and } \\
\text { physical examination } \\
\text { - Assessment function } \\
\text { and activity limitations } \\
\text { - Setting mutual goals }\end{array}$ & $\begin{array}{l}\text { - Radiological assessment } \\
\text { - Assessment of pain coping } \\
\text { and psychosocial factors } \\
\text { - Adjust goals }\end{array}$ & $\begin{array}{l}\text { - Consultation specialist } \\
\text { - Adjust goals }\end{array}$ \\
\hline Treatment modalities & $\begin{array}{l}\text { - Education } \\
\text { - Lifestyle advice } \\
\text { - Medication } \\
\text { - Acetaminophen } \\
\text { - Glucosaminesulphate }\end{array}$ & $\begin{array}{l}\text { - Exercise therapy } \\
\text { - Dietary therapy } \\
\text { - Medication }{ }^{\mathrm{b}} \\
\text { - (Topical) NSAIDs } \\
\text { - Tramadol }\end{array}$ & $\begin{array}{l}\text { - Multidisciplinary care } \\
\text { - TENS } \\
\text { - Medication } \\
\text { - Intra-articular injections }\end{array}$ \\
\hline Evaluation & - After 3 months ${ }^{\mathrm{c}}$ & - After 3-6 months ${ }^{\mathrm{c}}$ & - Patient sets interval \\
\hline
\end{tabular}

${ }^{\mathrm{a}}$ If there is a discrepancy between medical history and physical examination

${ }^{\mathrm{b}}$ Consult current guidelines for an adequate dose [1,27]

${ }^{\mathrm{c}}$ Or earlier, if the symptoms persist or increase

utilization and timing of different options. Moreover, the stepped care strategy endorses quality care as it is built on evidence-based treatment options for patients with hip or knee OA. With this instrument, the different health care providers involved in managing $\mathrm{OA}$ and the patients with hip or knee OA can improve their communication with each other.

A possible limitation of our study is that we did not apply a generally accepted consensus technique. However, our consensus procedure resembles the nominal group technique and includes important characteristics of good consensus methods such as face-to-face meetings, balanced group composition, feedback, iteration and involvement of experts [29]. Instead of ranking, all differences in opinions and raised concerns were resolved by discussion in multiple rounds and after multiple consultations of experts and patient representative. In our opinion, the used extensive procedure warrants the quality of content of the proposed stepped care strategy.

Although the stepped care strategy is based on the Dutch multidisciplinary guideline, the approach is likely to be also applicable for other countries, as recommendations in various national and international guidelines are very similar. In case of discrepancies, the proposed stepped care strategy can be easily adjusted. For example, in contrast to the Dutch guideline [1], the National Institute for Health and Clinical Excellence (NICE) guideline [4] recommends braces and capsaicin which could be included in the stepped care strategy.

A possible drawback for such a stepped care approach is that all patients are initially offered treatment options proposed in the first step of the model, while more advanced treatment options, such as joint surgery, may be more appropriate for patients with advanced OA. However, treatment options proposed in later steps could be offered, if considered appropriate. Also, it has been reported that almost half of the patients referred to secondary care because of OA benefited from a stepped care approach and no predictors for response to non-surgical treatment options could be identified [10]. Therefore, in our opinion, despite the risk for delay of more advanced treatment, the stepped care strategy is a suitable approach for patients with knee or hip OA.

We believe that a successful implementation of the stepped care strategy. BART will improve the outcome and quality of non-surgical care for patients with hip or knee OA substantially. Moreover, it will improve the communication between the patient and the different health care providers. At this moment, we are implementing BART strategy in a region in the Netherlands, and will identify barriers and facilitators for implementing. Therefore, a number of activities will be employed to inform the relevant health care providers about the content of the stepped care strategy, like implementation of a care booklet, newsletters, scientific articles, meetings and (periodical) education. Regional working groups play an important role in contacting and informing their adherents about the stepped care strategy. Most activities will be performed in primary care setting.

To facilitate implementation the booklet "Care for Osteoarthritis" (Zorgwijzer Artrose ${ }^{\circledR}$ ) is developed. Besides providing a patient-friendly version of the stepped care strategy, this booklet contains concise tools to enhance the patient's active role and communication between health care providers and the patient, such as leaflets to monitor symptoms, prepare consultations with health care providers, and draw up a comprehensive overview of the treatment options that the patient has already tried.

In summary, we developed a stepped care strategy to optimize the utilization and timing of the non-surgical 
treatment options for patients with osteoarthritis of hip or knee. Because osteoarthritis is a heterogeneous and longstanding disease, possible benefits of the proposed model of care will only pay off in the long term. Therefore, long-term prospective studies are needed to evaluate its cost-effectiveness.

Acknowledgements The authors are indebted to the participants of the written consultation and invitational conference for their contribution in the development of the stepped care strategy. Our thanks go to Hans Bloo, Roessingh Research \& Development, Enschede; Alfons den Broeder, Department of Rheumatology, Sint Maartenskliniek, Nijmegen; Sjoerd Bulstra, Department of Orthopaedics, University Medical Center Groningen; Martin van der Esch, Department of Rehabilitation and Rheumatology, Reade, Amsterdam; Wim Hilberdink, Paramedical center for rheumatology and rehabilitation, Groningen; Ria Jansen, Patient Society Poly-Osteoarthritis Companions, Hoogerheide; Caroline Kielstra, Bone and Joint Decade NL, Leiden; Margreet Kloppenburg, Department of Rheumatology, University Medical Center, Leiden; Marianne Krijgsman, Dutch patient organization for rheumatic diseases, Amersfoort; Annet Lievense, Department of General Practice, Erasmus University Medical Center Rotterdam; Sjoerd Olthof, Royal Dutch Society for Physical Therapy, Utrecht; Rob Oostendorp, Dutch Institute of Allied Health Care, Amersfoort; Wim Opstelten, The Dutch College of General Practitioners, Utrecht; Susan Picavet, National Institute for Public Health and the Environment, Bilthoven; Ruud Pöll, EMGO Institute for Health and Care Research VU University Medical Center, Amsterdam; Victor Pop, Faculty of Social and Behavioural Sciences, Tilburg University; Peter van Roermund, Bone and Joint Decade NL, Leiden; Marieke Scholte, Bone and Joint Decade NL, Leiden; Rosalinda Schuil-Menninga, Paramedical center for rheumatology and rehabilitation, Groningen; Gijs Snijders, Department of Rheumatology, Sint Maartenskliniek, Nijmegen; Cindy Veenhof, Netherlands Institute for Health Services Research, Utrecht; Cora Verberk; Gust Verbruggen, Department of Rheumatology, Ghent University Hospital, Belgium; Jan Verhaar, Department of Orthopaedics, Erasmus University Medical Center Rotterdam; Kees Verheijen, Department of Orthopaedics, Isala clinics, Zwolle; Geert Walenkamp, Department of Orthopaedics, Maastricht University Medical Center, Research Institute Caphri.

Disclosures None.

\section{References}

1. Dutch Orthopadic Association (2007) Diagnostiek en behandeling van heup- en knieartrose. Diagnostics and treatment of osteoarthritis of the hip and knee. Kwaliteitsinstituut voor de Gezondheidszorg (CBO). http://www.cbo.nl/Downloads/363/rl_heup_knie_07.pdf

2. Zhang W, Doherty M, Arden N, Bannwarth B, Bijlsma J, Gunther $\mathrm{KP}$ et al (2005) EULAR evidence based recommendations for the management of hip osteoarthritis: report of a task force of the EULAR Standing Committee for International Clinical Studies Including Therapeutics (ESCISIT). Ann Rheum Dis 64(5):669-681

3. Zhang W, Moskowitz RW, Nuki G, Abramson S, Altman RD, Arden N et al (2007) OARSI recommendations for the management of hip and knee osteoarthritis: Part I. Critical appraisal of existing treatment guidelines and systematic review of current research evidence. Osteoarthritis Cartil 15(9):981-1000

4. National Collaborating Centre for Chronic Conditions (2008) Osteoarthritis: national clinical guideline for care and management in adults. Royal College of Physicians, London

5. Swierstra BA, Bijlsma JWJ, Beer de JJA, Kuijpers T (2009) Richtlijn 'Diagnostiek en behandeling van heup- en knieartrose'.
(Guideline 'Diagnostics and treatment of osteoarthrosis of the hip and knee'). Ned Tijdschr Geneeskd 153(B39 (Richtlijnen))

6. Shrier I, Feldman DE, Gaudet MC, Rossignol M, Zukor D, Tanzer $\mathrm{M}$ et al (2006) Conservative non-pharmacological treatment options are not frequently used in the management of hip osteoarthritis. J Sci Med Sport 9(1-2):81-86

7. DeHaan MN, Guzman J, Bayley MT, Bell MJ (2007) Knee osteoarthritis clinical practice guidelines - how are we doing? J Rheumatol 34(10):2099-2105

8. Mitchell HL, Hurley MV (2008) Management of chronic knee pain: a survey of patient preferences and treatment received. BMC Musculoskelet Disord 9:123

9. Glazier RH, Dalby DM, Badley EM, Hawker GA, Bell MJ, Buchbinder R et al (1998) Management of common musculoskeletal problems: a survey of Ontario primary care physicians. CMAJ 158 (8): $1037-1040$

10. Snijders GF, den Broeder AA, van Riel PL, Straten VH, de Man FH, van den Hoogen FH et al (2011) Evidence-based tailored conservative treatment of knee and hip osteoarthritis: between knowing and doing. Scand J Rheumatol 40(3):225-231

11. Jamtvedt G, Dahm KT, Christie A, Moe RH, Haavardsholm E, Holm I et al (2008) Physical therapy interventions for patients with osteoarthritis of the knee: an overview of systematic reviews. Phys Ther 88(1):123-136

12. Grol R, Grimshaw J (2003) From best evidence to best practice: effective implementation of change in patients' care. Lancet 362 (9391): $1225-1230$

13. Brand C, Cox S (2006) Systems for implementing best practice for a chronic disease: management of osteoarthritis of the hip and knee. Intern Med J 36(3):170-179

14. Hunter DJ (2010) Quality of osteoarthritis care for communitydwelling older adults. Clin Geriatr Med 26(3):401-417

15. Hunter DJ, Neogi T, Hochberg MC (2011) Quality of osteoarthritis management and the need for reform in the US. Arthritis Care Res (Hoboken) 63(1):31-38

16. Williams B, Poulter NR, Brown MJ, Davis M, McInnes GT, Potter JF et al (2004) Guidelines for management of hypertension: report of the fourth working party of the British Hypertension Society, 2004-BHS IV. J Hum Hypertens 18(3):139-185

17. Von Korff M, Tiemens B (2000) Individualized stepped care of chronic illness. West J Med 172(2):133-137

18. McFarland KF (1997) Type 2 diabetes: stepped-care approach to patient management. Geriatrics 52(10):22-26, 35, 39

19. Katon W, Von Korff M, Lin E, Simon G, Walker E, Unutzer J et al (1999) Stepped collaborative care for primary care patients with persistent symptoms of depression: a randomized trial. Arch Gen Psychiatry 56(12):1109-1115

20. Von Korff M (1999) Pain management in primary care: an individualized stepped care approach. In: Gatchel R, Turk D (eds) Psychosocial factors in pain: evolution and revolutions. Guilford Press, New York, NY, pp 260-373

21. Porcheret M, Jordan K, Croft P (2007) Treatment of knee pain in older adults in primary care: development of an evidence-based model of care. Rheumatology (Oxford) 46(4):638-648

22. van den Ende CM, Bierma-Zeinstra SM, Vlieland TP, Swierstra BA, Voorn TB, Dekker J (2010) Conservatieve behandeling van heup- en knieartrose: Systematische en stapsgewijze behandelstrategie. (Conservative treatment of hip and knee osteoarthritis: a systematic, step-by-step treatment strategy). Ned Tijdschr Geneeskd 154:A1574

23. Bijlsma JW, Knahr K (2007) Strategies for the prevention and management of osteoarthritis of the hip and knee. Best Pract Res Clin Rheumatol 21(1):59-76

24. Doherty M, Dougados M (2001) Evidence-based management of osteoarthritis: practical issues relating to the data. Best Pract Res Clin Rheumatol 15(4):517-525 
25. Farley FA, Weinstein SL (2006) The case for patient-centered care in orthopaedics. J Am Acad Orthop Surg 14(8):447-451

26. Bijl D, Dirven-Meijer PC, Opstelten W, Raaijmakers AJ, Scholten RJPM, Eizenga WH et al (1998) NHG-Standaard: NietTraumatische Knieproblemen bij Volwassenen. (NHG-Standard: Non-traumatic knee complaints in adolescents). Huisarts Wet 41 (7):344-350

27. Belo JN, Bierma-Zeinstra SM, Raaijmakers AJ, Van der Wissel F, Opstelten W (2008) NHG Standaard Niet traumatische kniepro- blemen bij volwassenen (eerste herziening). (NHG-Standard: Non-traumatic knee complaints in adolescents (first revision)). Huisarts Wet 51(5):229-240

28. Vogels EMHM, Hendriks HJM, Van Baar ME, Dekker J, HopmanRock M, Oostendorp RAB, et al. (2005) KNGF-richtlijn Artrose heup-knie. (KNGF-guideline Osteoarthritis hip-knee). Neder Tijdschr Fysiother 1(supplement): 115

29. Campbell SM, Cantrill JA (2001) Consensus methods in prescribing research. J Clin Pharm Ther 26(1):5-14 